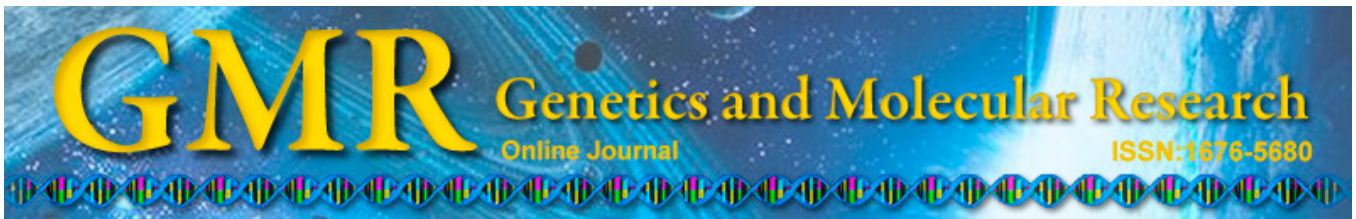

\title{
Characterization of a novel anther-specific gene encoding a leucine-rich repeat protein in petunia
}

\author{
Y.Z. Yue, J. Sun, X. Huang, H. Peng, G.F. Liu and H.R. Hu \\ Key Laboratory of Horticultural Plant Biology (Ministry of Education), \\ College of Horticulture and Forestry Sciences, \\ Huazhong Agricultural University, Wuhan, Hubei Province, China \\ Corresponding author: H.R. Hu \\ E-mail: huhuirong@mail.hzau.edu.cn
}

Genet. Mol. Res. 13 (4): 9889-9898 (2014)

Received October 28, 2013

Accepted March 17, 2014

Published November 27, 2014

DOI http://dx.doi.org/10.4238/2014.November.27.17

ABSTRACT. In Petunia x hybrida 'Fantasy Red', a leucine-rich repeat (LRR) gene referred to as $P h L R R$, was identified in a flower bud cDNA library. The open reading frame sequence of $P h L R R$ was $1251 \mathrm{bp}$, encoding a putative $46.2-\mathrm{kDa}$ protein of 416 amino acids. The PhLRR protein showed high similarity to members of polygalacturonase inhibitor proteins (PGIPs), contained 11 conserved LRR domains, and was an extracellular localization protein. Phylogenetic analysis showed that PhLRR belonged to the same PGIPs subfamily as SHY, indicating that PhLRR may be involved in the development of pollenlike $S H Y$. Expression analysis revealed that $P h L R R$ was abundantly expressed during early stages of flower bud and anther development, while it was not detected in any other examined organs, such as sepals, petals, pistils, roots, stems, leaves, or open flowers. Furthermore, many cis-acting elements (such as AGAAA and GTGA) related to anther-specific gene expression were identified in the $P h L R R$ gene promoter region, indicating that the promoter is also anther-specific. These results suggested that $P h L R R$ is a novel anther-specific gene 
that may be essential for the early development of anthers.

Key words: Petunia x hybrida 'Fantasy Red'; Anther-specific gene; Quantitative real-time RT-PCR; Promoter region

\section{INTRODUCTION}

Anthers form during a specific period of flowering plant life cycles, carrying pollens, which plays a key role in plant reproduction. Recently, several anther-specific genes related to pollen development have been identified in various plant species through high-throughput genomic studies (Kato et al., 2010; Li et al., 2010; El-Shehawi et al., 2011; Viswanathan et al., 2011). Moreover, the production of fertile pollen depends on a complex regulatory network, as expression of anther/pollen-specific genes may play vital roles in this process, and disruption during development of anther/pollens can result in male-sterile plants (Glover et al., 1998). Interestingly, various cis-acting regulatory/enhancer elements involved in anther/ pollen-specific expression have been identified in the promoters of anther/pollen-specific genes, and the $5 \phi$ upstream regions of anther/pollen-specific genes typically exhibit tissuespecific functions (Swapna et al., 2011; Chen et al., 2010, 2012b; Khurana et al., 2013).

Petunia hybrida is one of the most important ornamental plants, and is also a model material for the study of plant development biology. Many anther/pollen-specific genes have been identified through PCR-based screening of organ-specific cDNA libraries in petunia (Kobayashi et al., 1998; Cnudde et al., 2006). However, specific anther development-related genes have not been fully identified or characterized, and the molecular mechanism of anther development is largely unknown in petunia.

Leucine-rich repeat (LRR)-containing proteins from plants have diverse overall structures and functions. The largest group in the LRR superfamily include the adhesive proteins (Kobe and Deisenhofer, 1995). The structure of the PhLRR protein is nearly identical to those of polygalacturonase inhibitor proteins (PGIPs). PGIPs have been identified as defense proteins as their activity increases in response to wounding, elicitors, and fungal infection (De Lorenzo et al., 2001). However, PGIPs of plants may not always function as defense proteins; they also participate in developmental activities. The SHY protein, which plays an important role in mediating pollen tube growth, functions in a signal transduction pathways (Guyon et al., 2004).

In the present study, PhLRR, an anther-specific gene containing LRRs, was isolated from flower buds. Expression analysis and promoter cis-acting element prediction suggested that $P h L R R$ is an anther-specific gene expressed in flower buds during initial developmental stages. This is the first report regarding the expression and functional analysis of the PhLRR gene in plants. Studies examining PhLRR may further our understanding of the molecular mechanisms of anther development in petunia.

\section{MATERIAL AND METHODS}

\section{Plant materials}

Petunia plants (Petunia x hybrida 'Fantasy Red') were grown in the experimental 
field of Huazhong Agricultural University, Wuhan, China. Materials for RNA extraction were sampled from at least 5 individual flowering plants and mixed randomly. RNA was extracted from the flower buds and anthers at 8 developmental stages [bud1 $(\mathrm{Bl}<2 \mathrm{~mm})$, bud2 (B1 3 $\pm 0.5 \mathrm{~mm}$ ), bud3 (Bl $5 \pm 0.5 \mathrm{~mm})$, bud4 (B1 $10 \pm 0.5 \mathrm{~mm})$, bud5 (Bl $15 \pm 0.5 \mathrm{~mm})$, bud6 (B1 $20 \pm 0.5 \mathrm{~mm}$ ), bud7 (B1 $25 \pm 0.5 \mathrm{~mm}$ ), and bud8 (B1 $35 \pm 0.5 \mathrm{~mm})]$ (Bl indicates the bud length excluding sepal), and from roots, tender stems, fresh leaves, and opening flowers, as well as from 4 whorls of floral organs (sepal, petal, anther, and pistil) in bud2, for use in realtime reverse transcriptase (RT)-polymerase chain reaction (PCR) analysis. Upon harvesting, these materials were immediately frozen in liquid nitrogen and stored at $-80^{\circ} \mathrm{C}$ until RNA was extracted.

\section{DNA/RNA extraction and cDNA synthesis}

Genomic DNA was extracted from young petunia leaves using the CTAB method (Murray and Thompson, 1980). Total RNA was extracted from flower buds, anthers, and other tissues using RNAiso Reagent (TaKaRa, Shiga, Japan) according to previously described methods (Yue et al., 2013). RNA quality was evaluated by electrophoresis on $1.2 \%$ denaturing agarose gels. First-strand cDNA was synthesized using a TransScript One-Step gDNA Removal and cDNA Synthesis SuperMix kit (TransGene, Beijing, China) according to manufacturer instructions.

\section{Isolation of $P h L R R$ and its promoter}

Expressed sequence tags (ESTs) of PhLRR were acquired from the library by suppression subtractive hybridization. Through the EST BLAST in the genome database of Petunia hybrida, we successfully predicted the full-length open reading frame (ORF) of PhLRR and the promoter region using the FGENESH tool (http://www.softberry.com). The full-length cDNA and gDNA were amplified using the primers F (5'-GCAGGAAGTTGGCAAAAGATG-3') and R (5'-GTCGCTCAGATAGAAGAAATAAGGC-3'). The primers F (5'-GCGGTTTGAAC TATTGATTTACG-3') and R (5'-TTTGTTTAGACAGCTTTTGAGTGAC-3') were used to clone the promoter region of the PhLRR ATG start codon from petunia genomic DNA. These primers were all designed using the Primer 5 software.

\section{Sequence analysis}

Sequence character prediction of PhLRR was conducted using the ProtParam tool (http://www.expasy.ch/tools/protparam.html). Hydrophobicity of PhLRR was analyzed using the ProtScale tool (http://web.expasy.org/protscale/). A phylogenetic tree was constructed using MEGA5 based on the neighbor-joining method with 1000 bootstrap replications (Kumar et al., 2004). Amino acid sequences used in phylogenetic tree construction were obtained from NCBI (http://www.ncbi.nlm.nih.gov/). Sequence of amino acid sequences were aligned using the ClustalW method (www.ebi.ac.uk/clustalw). The putative domain was predicted by Simple Modular Architecture Research Tool (SMART) (http://smart.embl-heidelberg.de/). NetNGlyc 1.0 Server and NetPhos 2.0 Server were used to predict the N-linked glycosylation sites and phosphorylation sites. PLACE (http://www.dna.affrc.go.jp/PLACE/signalup.html) was used to search for putative cis-acting elements in the promoter region. 


\section{Expression analysis by quantitative real-time RT-PCR}

Quantitative real-time RT-PCR (qRT-PCR) was performed using an ABI 7500 Fast Sequence Detection System (PE Applied Biosystems, Foster City, CA, USA). The primers F (5'-GAAGGAGATGAATTGGAGGGG-3') and R (5'-CATTAAAAAG

ACTGCCAGAGAAAAA-3') were designed using the Primer Premier 5 software. Beta-Actin was used as an internal control, and a negative control (no template) was included in each run. Reactions were performed using the SYBR ${ }^{\circledR}$ Premix Ex Taq ${ }^{\mathrm{TM}}$ (TaKaRa). Briefly, PCR products were amplified using $1 \mu \mathrm{L}$ template from the RT reaction mixture, $5 \mu \mathrm{L} 2 \mathrm{X}$ SYBR ${ }^{\circledR}$ Premix Ex Taq ${ }^{\mathrm{TM}}, 0.5 \mu \mathrm{L}$ of each forward and reverse primer $(10 \mu \mathrm{M})$, and water to a final volume of $20 \mu \mathrm{L}$. The thermal cycling conditions were programmed based on previously described methods (Yue et al., 2013). A melting temperature cycle was performed to confirm the presence of a single product. qRT-PCR was performed in 3 replicates for each sample, with the data shown as means $\pm \mathrm{SD}(\mathrm{N}=3)$.

\section{RESULTS}

\section{Cloning of PhLRR}

To identify new genes encoding proteins that regulate flower bud development in petunia plants, we constructed a suppression subtractive hybridization cDNA library. A cDNA fragment of $663 \mathrm{bp}$ appeared to accumulate specifically in the flower bud. The sequence BLAST results (BLASTX) showed that the putative amino acid sequence encoded by this fragment was homologous to LRR proteins. Thus, EST BLAST of the genome database of Petunia hybrida successfully predicted the full-length ORF of the LRR protein using the FGENESH tool (http://www.softberry.com). A sequence with putative ORFs were obtained using RT-PCR and designated as PhLRR (GenBank accession No. KF146986).

\section{Sequence analysis}

The 1355-bp PhLRR cDNA contains a 1251-bp ORF, encoding a 416-amino acid protein (Figure 1). The deduced molecular mass of the mature PhLRR protein was $46.2 \mathrm{kDa}$, with a predicted $\mathrm{pI}$ of 7.08 . The amino acid sequence displayed 17 possible phosphorylation sites and 4 putative glycosylation sites for N-linked glycosyl chains (NXS/T, where X denotes any amino acid).

The region spanning from amino acids 90-344 contained 11 tandem repeats with an average size of 24 amino acids: 13 of the 24 residues were found to be conserved (Figure 2). The composition of the repeats was very similar to the LRR motif, which was observed in several other proteins (Figure 2). Alignment of LRRs revealed a 24-amino acid repeat matching the external LRR consensus (Figure 2), LxxLxxLxxLxLxxNxLxGxIPxx, as observed in the tomato Cf-2 and Cf-9 genes (Jones and Jones, 1997). Using GenomeNet CLUSTALW, multiple sequence alignment was obtained for 14 accessions of the PhLRR/ PGIP/LRP/SLRP gene family. Phylogenetic analysis showed that different types of LRR proteins were classified into the same groups. PhLRR and SHY were clustered in the same clade (Figure 3). 


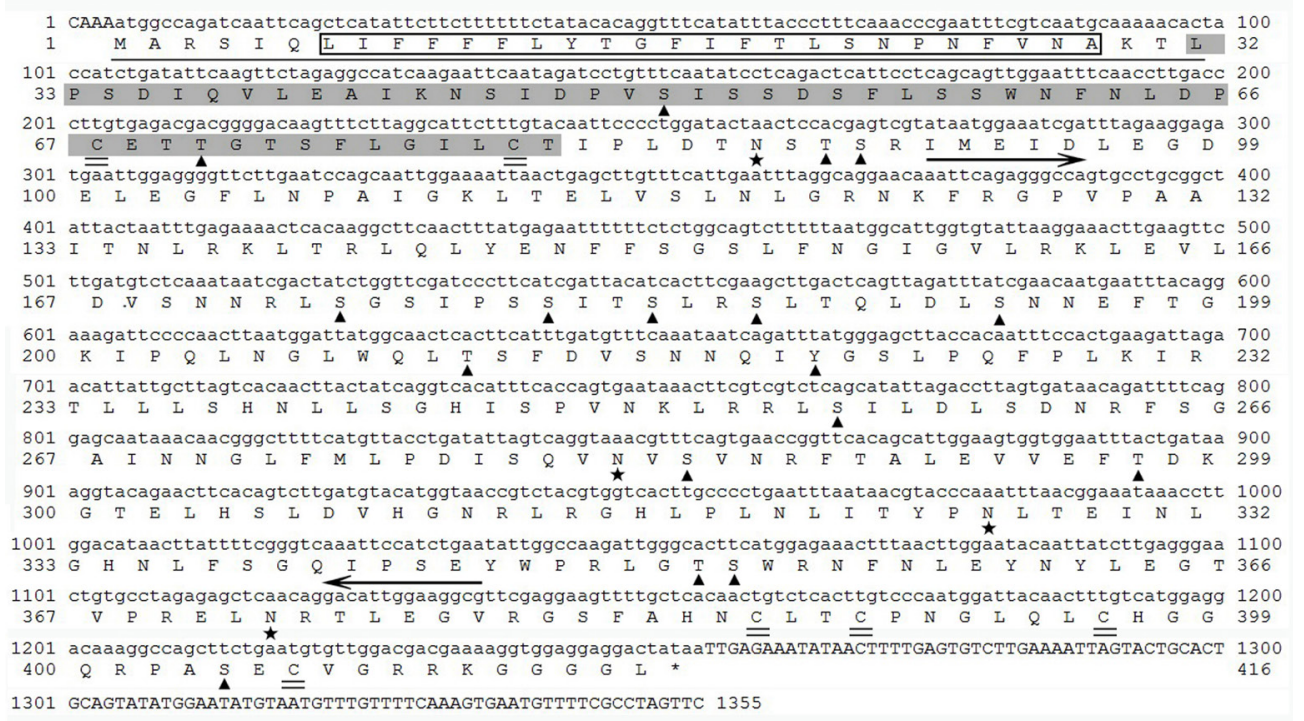

Figure 1.cDNA and deduced amino acid sequence of the $P h L R R$ gene. The small asterisk at position 1253 indicates the translation termination codon. The putative signal peptide for secretion is underlined. Putative N-glycosylation sites and the phosphorylation sites are shown by black triangles and by big asterisks, respectively. The putative leucine zipper domain is represented by shaded box. The predicted transmembrane sequence is boxed. The LRR region is marked by arrows.

\section{Expression analysis of PhLRR}

Expression analysis was performed to examine the space and time expression profile of PhLRR. In flower buds in different stages, this gene was highly amplified in bud1 to bud2 and largely decreased in bud3 (Figure 4). PhLRR was found to be expressed only in anthers, but not detected in sepals, petals, pistils, roots, tender stems, fresh leaves, or open flowers (Figure 5). Since the anther is only a small component of the petunia flower organ, particularly in stages bud4 to bud8, the expression of this gene may not be detected during late-stage flower buds. Hence, to explore the expression of this gene in different stages of anther development, anthers (an1 to an8) were stripped from bud1 to bud8, respectively, and subjected to time expression analysis. The results showed that PhLRR was highly expressed during the initial stages of anther development (Figure 6).

\section{Promoter analysis of $P h L R R$}

$P h L R R$ was particularly abundant in anthers. To gain further insight into the regulation mechanism of PhLRR expression, a 1254-bp promoter upstream of the PhLRR ATG start codon from petunia genomic DNA was cloned (Figure 7). Various regulatory motifs (Swapna et al., 2011) related to anther/pollen-specific expression were observed in the PhLRR promoter region, such as ACGT MOTIF (ACGT), DOFCOREZM (AAAG), POLLENILELAT52 (AGAAA), GTGA MOTIF (GTGA), LAT enhancer element (TGTGA, TGTGG), and LAT52 quantitative element (TGGTTA) (Figure 7). 
A

\begin{tabular}{|c|c|}
\hline \multicolumn{2}{|r|}{. . . . . I IMEI DLEGDELEG. FLNPA } \\
\hline & I TNL RKL TRLQL YENF F SG. SLF NG \\
\hline & I GVLRKLEVLDVSNNRLSG. SI PSS \\
\hline & I TSLRSL TQLDLSNNEF TG. KI PQ. \\
\hline & LNGL WQL TSFDVSNNQI YG. SLPQF \\
\hline & . . . PLKI RTLLLSHNLLSG. HI SP. \\
\hline & VNKL RRL SI LDL SDNRF S G. AI NNG \\
\hline & LFNLPDI SQVNVS VNRF TALEVVEF \\
\hline & TDKGTELHSLDVHGNRL RG. HLPLN \\
\hline & LI TYPNL TEI NLGHNLFSG. QI PSE \\
\hline & Lx $x$ Lx $\times L \times x$ LDLS $\times N \times F S G \times I P x \times$ \\
\hline
\end{tabular}

B

PROTEIN CONSENSUS SEQUENCE LRRS REFERENCE LXXLXXXXLDLXXNLLGXI PXX

\begin{tabular}{|c|c|c|c|}
\hline & LxxLXXLXLDLSXNXFSGXIPXx & 11 & Yue, this work \\
\hline BRI1 & FxxFxxLXXLLLSNXFSGXIPXX & 25 & Li and Chory, 1997 \\
\hline CALRR1 & LxxLxxLxxLxLxxNxLxGXIPxx & 5 & Jung et al., 2004 \\
\hline Cf-2.1 & LGNL $x$ x $x \times L x L x \times N$ xL xGSI Pxx & 36 & Dixon et al., 1996 \\
\hline Cf-9 & LXXLXXLXIDLSSNNLXGXIPSX & 28 & Jones et al., 1994 \\
\hline CLAVATA 1 & FXxFXXLXXLXFXXNXFGXIPXX & 23 & Clark et al., 1997 \\
\hline FIL2 & LXXLXXLXSLXLSXNXLXGXI PXX & 10 & Steinmayr et al., 1994 \\
\hline LRPKm1 & LxxLxxLxLDLxxNLSGxI Pxx & 23 & Komjanc et al., 1999 \\
\hline $\mathrm{N}$ & $x \times x L \times x L x \operatorname{LDL} x \times x \times L \times x L P x L$ & 14 & Whitham et al., 1994 \\
\hline NtLRR1 & LxxLxxLxxLxLxNxLxGxIPxx & 9 & \\
\hline NtLRR2 2 & LxxLxxLxxLxLxxNxLxGXI Pxx & 3 & Xu et al., 2009 \\
\hline PGIP(bean) & LxxLxxLxxLDLS $x N \times L \times G \times L P x x$ & 10 & De Lorenzo et al., 1994 \\
\hline RLK5 & 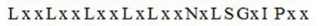 & 21 & Walker et al., 1993 \\
\hline RPM1 & PxxLxxLxxLDL. $x \times x \times L x \times L P x L$ & 8 & Grant et al., 1995 \\
\hline RPS2 & 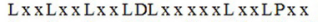 & 11 & Bent et al., 1995 \\
\hline SHY & LxxLxxLxxLxLxNXLxGxIPxS & 10 & Guyon et al., 2004 \\
\hline $\mathrm{Xa} 21$ & LXXLXX $x \times$ LDLSSNNL $x$ GXI Pxx & 23 & Song et al., 1995 \\
\hline ZmSERK1 & LGXLXXLXXLDLXNNNLSGXI PXX & 5 & Baudino et al., 2001 \\
\hline ZmSERK2 & LGNL $x$ x $\mathrm{Lx}$ L LDL $x$ NNNL S GXI Pxx & & Baudino et al., 2001 \\
\hline
\end{tabular}

Figure 2. Characteristics of the LRR region of PhLRR. A. Alignment of the 11 LRRs of PhLRR. Numbers to the right indicate the specific LRR number. The bottom line indicates the consensus sequence for the PhLRR LRR. " $\mathrm{x}$ " stands for any amino acid. B. Comparison of the LRR consensus sequence of PhLRR and other LRR proteins.

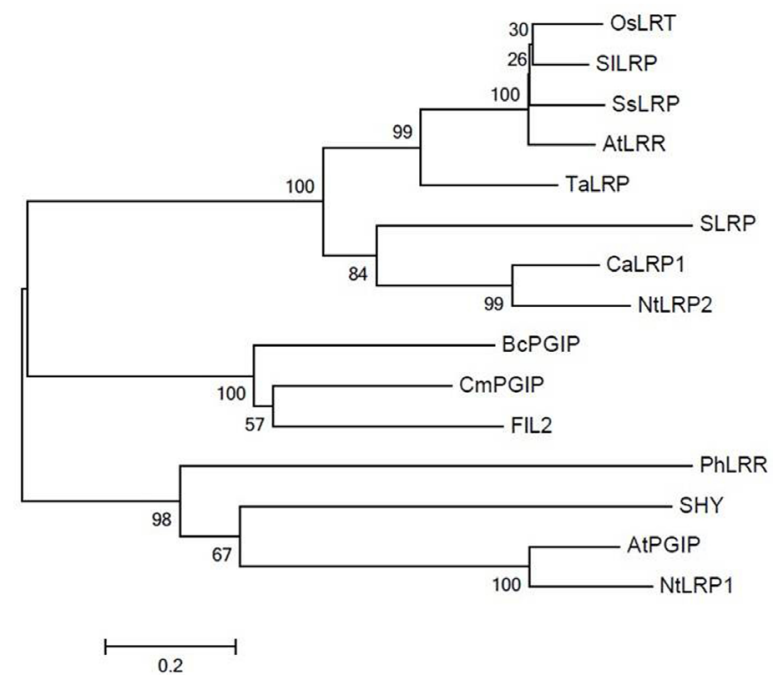

Figure 3. Unrooted phylogenetic tree of PhLRR with other LRR proteins. Phylogenetic tree was constructed by the MEGA5 software. Scale bar represents 0.2 substitutions per site. Numbers on the tree represents confidence values from bootstrap test for 1000 replicates. The accession number of each selected LRR protein is as follows: FIL2: CAA54303; SHY: AF325673; NtLRP1: DQ358108; SLRP: AAC49559; CaLRP1: AAN62015; NtLRP2: EF535611; TaLRP: AAU82111; SsLRP: FJ787729; SILRP: CAA64565; OsLRT: NP_001044578; AtLRR: NP_197608; BcPGIP: AAX68500; CmPGIP: AAP41199; AtPGIP: BAB02490. 


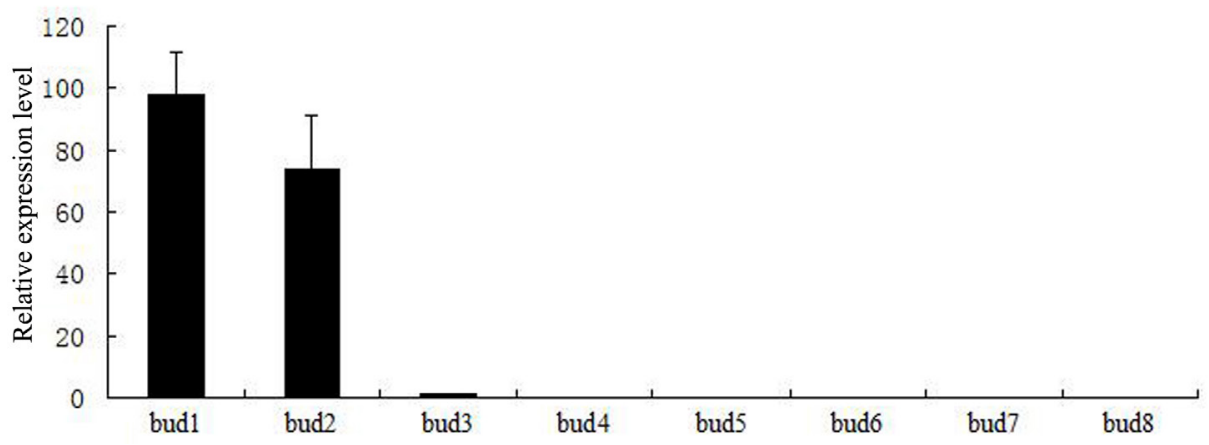

Figure 4. Real-time RT-PCR analysis of the PhLRR gene during the flower bud development stages in Petunia $\mathrm{x}$ hybrida 'Fantasy Red'. RT-PCR was performed using RNA isolated from eight different flower bud development stages [bud1 $(\mathrm{Bl}<2 \mathrm{~mm})$, bud2 (B1 $3 \pm 0.5 \mathrm{~mm})$, bud3 $(\mathrm{Bl} 5 \pm 0.5 \mathrm{~mm})$, bud4 $(\mathrm{Bl} 10 \pm 0.5 \mathrm{~mm})$, bud5 $(\mathrm{Bl} 15 \pm 0.5 \mathrm{~mm})$, bud6 (B1 $20 \pm 0.5 \mathrm{~mm}$ ), bud7 (B1 $25 \pm 0.5 \mathrm{~mm})$, bud8 (B1 $35 \pm 0.5 \mathrm{~mm})$ ] (B1 means the bud length excluding sepal).

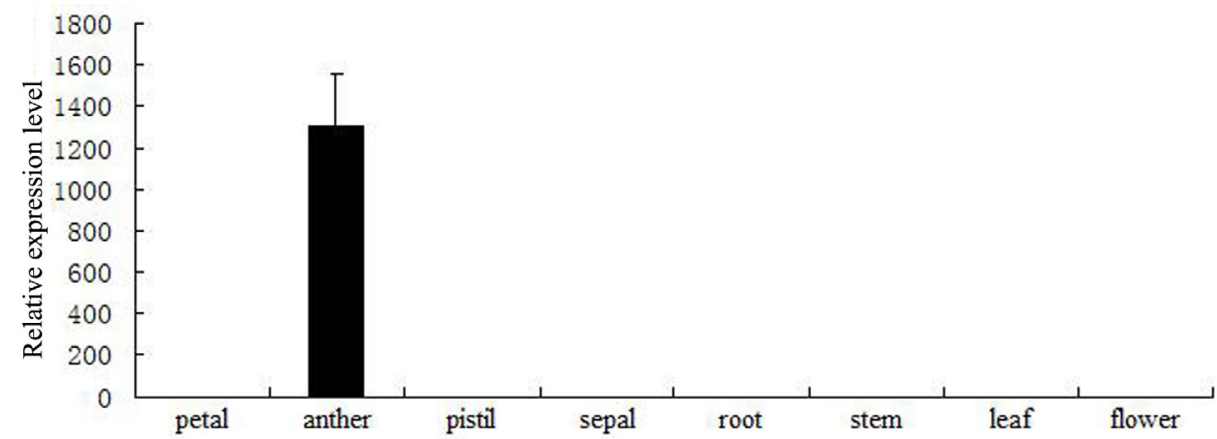

Figure 5. Analysis of the spatial expression pattern of the $P h L R R$ gene in different tissues (roots, tender stems, fresh leaves, open flowers and petal, anther, pistil, sepal from bud2) of the Petunia x hybrida 'Fantasy Red'.

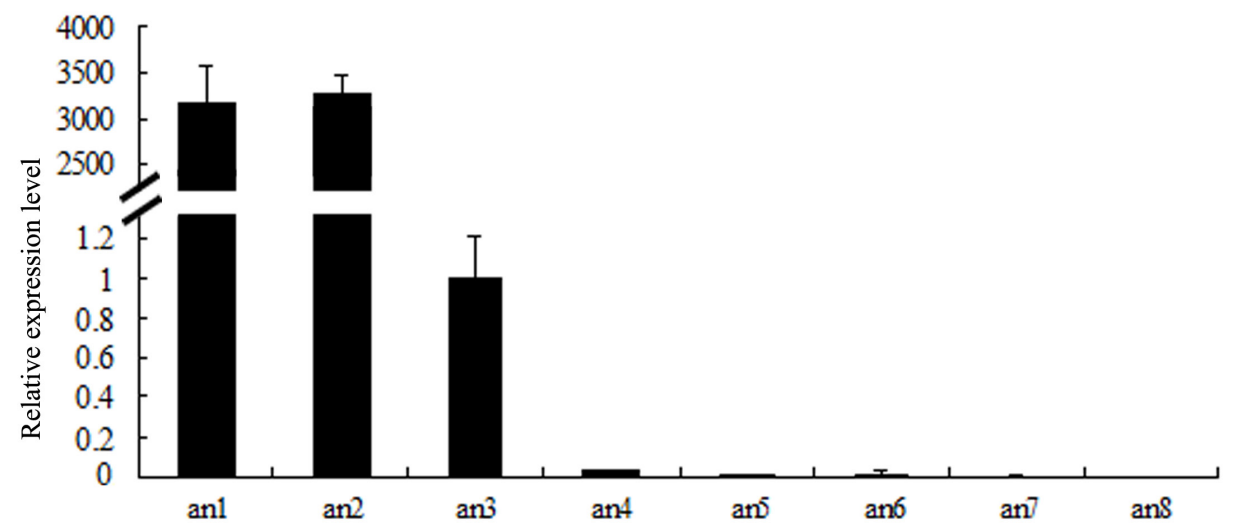

Figure 6. Real-time RT-PCR analysis of the PhLRR gene during the anther development stages in Petunia $\mathrm{x}$ hybrida 'Fantasy Red'. RT-PCR was performed using RNA isolated from eight different anther development stages (an1: $<2 \mathrm{~mm}$; an2: $3 \mathrm{~mm} \pm 0.5$; an3: $5 \mathrm{~mm} \pm 0.5$; an4: $10 \mathrm{~mm} \pm 0.5$; an5: $15 \mathrm{~mm} \pm 0.5$; an6: $20 \mathrm{~mm} \pm 0.5$; an 7: 25 $\mathrm{mm} \pm 0.5$; an $8: 35 \mathrm{~mm} \pm 0.5)$. an: anthers. 


\begin{tabular}{|c|c|}
\hline-1254 & $\begin{array}{l}\text { GCGGTTTGAACTATTGATTTACGTAATTGGAGGAGAAC } \\
\text { ACGT MOTIF }\end{array}$ \\
\hline-1204 & $\begin{array}{l}\text { CAAGAAATTCTGAAG TAAGGACTAATTTCCACTAGGCGATGTCGATTTAT } \\
\text { POLLENILELAT52 }\end{array}$ \\
\hline-1154 & CCTGGG TTTCTTATG TAAARATG TCACATCTAATATTTCCTAACCAC \\
\hline-1104 & CTTTGCAGTGCCGTARAAAAAARTTAA \\
\hline-1054 & CTCARACTTTATATATATGCTATARARTCTTCTTTACACTATTGTAT \\
\hline-1004 & AGTGTAATTTTGGATGAGAGCATCCAATTGAMTCCCACTCCTAGCATGT \\
\hline-954 & $\begin{array}{l}\text { GTGGCTCCGCCACTAG TACTACTAAGGCG TAATTTTTGTTG TT TCT TCAT } \\
\text { LAT enhancer element }\end{array}$ \\
\hline-904 & ATGGTTGCTTTAGATTCTTATTTTTCACTTCCTTTGTT \\
\hline-854 & \\
\hline-804 & GGATAGAGC \\
\hline-754 & GCACCTAG \\
\hline 704 & CGCABATTAMGTATATTATGTG \\
\hline-654 & TATGTCTAATTTGATGTTT \\
\hline-604 & CACTTACACTTAAAAAAATATGGATCAMT \\
\hline-554 & $\begin{array}{l}\text { TCTCATTTABATAAGGGCAATCCGG TGCACAAMGCATCATGCG TTCACTC } \\
\text { DOFCOREZM }\end{array}$ \\
\hline-504 & $\begin{array}{c}\text { AAGATCGGGAGAAGGACCCCACCCTTAGGGG TGTGATATATAGAGCCTAA } \\
\text { LAT enhancer element }\end{array}$ \\
\hline 454 & 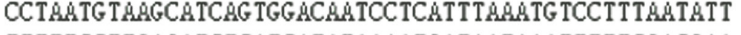 \\
\hline-404 & TTTTTCCTTGAGATCTCATCATATARAATGATASTAAATTTTTCGACGAS \\
\hline-354 & GGTTGTCTACTGACTACTTTTGGGATGCTGTAACTCCACTTCTAACG \\
\hline -304 & GTAAACTCATACTCTTAAAATTCTAGATCCTGACTCCGAGAGGAAT T \\
\hline-254 & CCGGATAAGATTAGCCAGGTTTACTAGAAGCCAACCACACATATCAGCTT \\
\hline-204 & TTACTTAT TATTAGGTTGGAACT TGGAACAGGACCAATGAAGCACTCTCT \\
\hline-154 & ACACTTGTAACCTACTTGCAHGAATCCATTTTGCATGACAATGCATGATC \\
\hline-104 & $\begin{array}{l}\text { ATTTTTTCCATGACCT TCCT AAAGTTTTCAACT TCTTACT TATGAAACT T } \\
\text { DOFCOREZM }\end{array}$ \\
\hline-54 & $\begin{array}{c}\text { CATATAAGGAGCTCTAACCAAATTATTCAGTCACTCAAAAGCTGTCTAAA } \\
\text { DOFCOREZM }\end{array}$ \\
\hline & Chas 目tggccagatcaattca \\
\hline
\end{tabular}

Figure 7. Analysis of putative cis-acting elements of the PhLRR promoter. The putative cis-acting elements (such as AGAAA, GTGA, TGTGA, TGTGG, TGGTTA, ACGT, and AAAG) are marked with single underlines. The first base of the translation start site is boxed and assigned as position +1 .

\section{DISCUSSION}

It is very important to investigate the genes that participate in pollen development, particularly those that are anther/pollen-specific ones. This information will not only increase the understanding of the molecular mechanism of sexual propagation in flowering plants, but may also be useful in agriculture for hybrid seed production.

$\mathrm{N}$-linked glycosylation was found to play a key role in protein folding, membrane targeting, and substrate binding (Xu et al., 2009). Several potential N-linked glycosylation sites (NXS/T) were found in the PhLRR protein (Figure 1), indicating that the PhLRR protein may be glycosylated post-translationally and then translocated to the cell wall and membranes. Additionally, many putative serine/threonine/tyrosine substrate residues were found in the PhLRR gene, supporting that PhLRR is phosphorylated post-translationally (Figure 1). Therefore, $P h L R R$ may be a receptor protein involved in early stages of signal transduction in the cell wall and plasma membranes and mediate protein interactions in various types of biological processes. Sequence analysis of $P h L R R$ using bioinformatic tools revealed that $P h L R R$ shares 
significant sequence similarity to some predicted LRR proteins, whereas the function or expression patterns of these genes remain unclear. Thus, we suggest that $P h L R R$ is a novel gene of unknown function. PhLRR contains a conserved LRR domain, which has a highly identity to PGIPs. The LRR domain is characterized by tandem arrays of a leucine-rich consensus sequence and a structurally conserved LRR interaction surface (Kobe and Deisenhofer, 1994; Ho et al., 2002). The PhLRR protein is composed of 11 tandem repeat LRR motifs (Figure 2), of which the diversity of LRR domains may be related to specific molecular characteristics.

According to phylogenetic analysis, PhLRR and SHY are clustered in the same clade with other genes encoding PGIPs (Figure 3). PGIPs, which play a role in plant defense and possibly during development, are also LRR proteins (Williams et al., 1992; Jones and Jones, 1997). $S H Y$ is a pollen-specific gene, encodes an LRR protein that has been further studied in petunia, and the structure of SHY protein is nearly identical to PGIPs of plants. However, experiments indicate that the SHY protein is not a defense protein that inhibits fungal endopolygalacturonases, suggesting that $S H Y$ functions in a signal transduction pathway controlling pollen tube growth (Guyon et al., 2004). Thus, PhLRR may also function in a signal transduction pathway and mediate plant anther/pollen development.

Recently, many anther/pollen-related or anther/pollen-specific genes have been isolated, characterized, and analyzed. Some of these genes were found to be expressed during early stages of male organ development (Zhang et al., 2011, 2012), some during late stages, and some during the entire period of male organ development (Robert et al., 1993; FourgouxNicol et al., 1999; Honys and Twell, 2004; Kato et al., 2010; Chen et al., 2012a). Expression analysis showed that $P h L R R$ was detected only in bud1-3, with expression peaking during the bud1 stage and then decreasing to a minimum during the 3rd stage. Similar expression patterns were observed during the various stages of anther development (Figures 4 and 6). Additionally, PhLRR was expressed at an extremely high level in the anthers of petunia, but was not detected in any other tissues (Figure 5). In our study, several different cis-element motifs involved in anther/pollen-specific expression were detected in the PhLRR promoter region (Figure 7), suggesting that this promoter may be an anther/pollen-specific promoter. Among the various motifs, ACGT and AAAG, which are the core binding sites of the sperm cell-specific AtGEX2 and OsGEX2 promoters, were shown to be target sequences of the bZIP DNA-binding regulatory proteins and Dof transcription factors (Williams et al., 1992; Yanagisawa and Schmidt, 1999). Remarkably, there were 7 copies of AAAG motifs and 1 copy of an ACGT motif in the PhLRR promoter region, indicating that expression of this gene is highly influenced by $b Z I P$ and Dof genes. These results imply that PhLRR is an anther-specific gene and plays a critical role in the initial stages of petunia anther development.

However, the precise physiological function of the $P h L R R$ gene in anther remains to be elucidated. Further studies examining PhLRR in petunia should be conducted using RNAi.

\section{ACKNOWLEDGMENTS}

Research supported by the Fundamental Research Funds for the Central Universities (Project \#2013PY085) and the Natural Science Foundation of Hubei Province of China (Project \#2014CFB926).

\section{REFERENCES}

Chen C, Liu S, Hao X, Chen G, et al. (2012a). Characterization of a pectin methylesterase gene homolog, CaPME1, 
expressed in anther tissues of Capsicum annuum L. Plant Mol. Biol. Rep. 30: 403-412.

Chen L, Tu Z, Hussain J, Cong L, et al. (2010). Isolation and heterologous transformation analysis of a pollen-specific promoter from wheat (Triticum aestivum L.). Mol. Biol. Rep. 37: 737-744.

Chen L, Miao Y, Wang C, Peipei Su, et al. (2012b). Characterization of a novel pollen-specific promoter from wheat (Triticum aestivum L.). Plant Mol. Biol. Rep. 30: 1426-1432.

Cnudde F, Hedatale V, de Jong H, Pierson ES, et al. (2006). Changes in gene expression during male meiosis in Petunia hybrida. Chromosome Res. 14: 919-932.

De Lorenzo G, D'Ovidio R and Cervone F (2001). The role of polygalacturonase-inhibiting proteins (PGIPs) in defense against pathogenic fungi. Anпи. Rev. Phytopathol. 39: 313-335.

El-Shehawi AM, Elseehy MM and Hedgcoth C (2011). Isolation and sequence analysis of wheat tissue-specific cDNAs by differential display. Plant Mol. Biol. Rep. 29: 135-148.

Fourgoux-Nicol A, Drouaud J, Haouazine N, Pelletier G, et al. (1999). Isolation of rapeseed genes expressed early and specifically during development of the male gametophyte. Plant Mol. Biol. 40: 857-872.

Glover J, Grelon M, Craig S, Chaudhury A, et al. (1998). Cloning and characterization of MS5 from Arabidopsis: a gene critical in male meiosis. Plant J. 15: 345-356.

Guyon V, Tang WH, Monti MM, Raiola A, et al. (2004). Antisense phenotypes reveal a role for SHY, a pollen-specific leucine-rich repeat protein, in pollen tube growth. Plant J. 39: 643-654.

Ho DN, Coburn GA, Kang Y, Cullen BR, et al. (2002). The crystal structure and mutational analysis of a novel RNAbinding domain found in the human Tap nuclear mRNA export factor. Proc. Natl. Acad. Sci. U. S. A. 99: 1888-1893.

Honys D and Twell D (2004). Transcriptome analysis of haploid male gametophyte development in Arabidopsis. Genome Biol. 5: R85.

Jones DA and Jones J (1997). The role of leucine-rich repeat proteins in plant defences. Adv. Bot. Res. 24: 89-167.

Kato H, Xie G, Sato Y and Imai R (2010). Isolation of anther-specific gene promoters suitable for transgene expression in rice. Plant Mol. Biol. Rep. 28: 381-387.

Khurana R, Kathuria H, Mukhopadhyay A, Kapoor S, et al. (2013). A 286 bp upstream regulatory region of a rice antherspecific gene, OSIPP3, confers pollen-specific expression in Arabidopsis. Biotechnol. Lett. 35: 455-462.

Kobayashi A, Sakamoto A, Kubo K, Rybka Z, et al. (1998). Seven zinc-finger transcription factors are expressed sequentially during the development of anthers in petunia. Plant J. 13: 571-576.

Kobe B and Deisenhofer J (1994). The leucine-rich repeat: a versatile binding motif. Trends Biochem. Sci. 19: 415-421.

Kobe B and Deisenhofer J (1995). Proteins with leucine-rich repeats. Curr. Opin. Struct. Biol. 5: 409-416.

Kumar S, Tamura K and Nei M (2004). MEGA3: Integrated software for Molecular Evolutionary Genetics Analysis and sequence alignment. Brief. Bioinform. 5: 150-163.

Li J, Yu M, Geng LL and Zhao J (2010). The fasciclin-like arabinogalactan protein gene, FLA3, is involved in microspore development of Arabidopsis. Plant J. 64: 482-497.

Murray MG and Thompson WF (1980). Rapid isolation of high molecular weight plant DNA. Nucleic Acids Res. 8: 43214325.

Robert LS, Allard S, Gerster JL, Cass L, et al. (1993). Isolation and characterization of a polygalacturonase gene highly expressed in Brassica napus pollen. Plant Mol. Biol. 23: 1273-1278.

Swapna L, Khurana R, Kumar SV, Tyagi AK, et al. (2011). Pollen-specific expression of Oryza sativa indica pollen allergen gene (OSIPA) promoter in rice and Arabidopsis transgenic systems. Mol. Biotechnol. 48: 49-59.

Viswanathan A, Kuriakose B, Bharadwaj S and Thomas G (2011). Expression of aprotinin in anther causes male sterility in tobacco var Petit havana. Plant Mol. Biol. Rep. 29: 825-834.

Williams ME, Foster R and Chua NH (1992). Sequences flanking the hexameric G-box core CACGTG affect the specificity of protein binding. Plant Cell 4: 485-496.

Xu ZS, Xiong TF, Ni ZY, Chen XP, et al. (2009). Isolation and identification of two genes encoding leucine-rich repeat (LRR) proteins differentially responsive to pathogen attack and salt stress in tobacco. Plant Sci. 176: 38-45.

Yanagisawa S and Schmidt RJ (1999). Diversity and similarity among recognition sequences of Dof transcription factors. Plant J. 17: 209-214.

Yue YZ, Ma FF, Huang X, Bao MZ, et al. (2013). Transcriptional profile of differentially expressed genes related to abortive flower buds under short light period stress in petunia. Sci. Hortic. 164: 323-332.

Zhang A, Chen Q, Huang L, Qiu L, et al. (2011). Cloning and expression of an anther-abundant polygalacturonase gene BcMF17 from Brassica campestris ssp. chinensis. Plant Mol. Biol. Rep. 29: 943-951.

Zhang A, Qiu L, Huang L, Yu X, et al. (2012). Isolation and characterization of an anther-specific polygalacturonase gene, BcMF16, in Brassica campestris ssp. chinensis. Plant Mol. Biol. Rep. 30: 330-338. 